\title{
Canine Carboxyl- and Hydroxyl-Containing Metabolites Altered after a Three-Week Supplementation of Cannabidiol (CBD)-Containing Treats in an Exploratory Study
}

\author{
Elizabeth M Morris ${ }^{1}$, Susanna E Kitts-Morgan², Dawn M Spangler², Ibukun M Ogunade ${ }^{3}$, Kyle R Mc Leod ${ }^{1}$, and David L Harmon ${ }^{1, *}$ \\ ${ }^{1}$ Department of Animal and Food Sciences, University of Kentucky, Lexington, United States \\ ${ }^{2}$ College of Veterinary Medicine, Lincoln Memorial University, Harrogate, United States \\ ${ }^{3}$ Division of Animal and Nutritional Science, West Virginia University, Morgantown, United States
}

*Corresponding author: David L Harmon, Department of Animal and Food Sciences, University of Kentucky, Lexington, United States, KY 40546; E-mail: david.harmon@uky.edu

Received: 04 Aug, 2021 | Accepted: 27 Aug, 2021 | Published: 08 Sep, 2021

Citation: Morris EM, Kitts-Morgan SE, Spangler DM, Ogunade IM, McLeod KR, et al. (2021) Canine Carboxyl- and Hydroxyl-Containing Metabolites Altered after a Three-Week Supplementation of Cannabidiol (CBD)-Containing Treats in an Exploratory Study. J Anim Sci Res 5(2): dx.doi. org/10.16966/2576-6457.153

Copyright: (c) 2021 Morris EM, et al. This is an open-access article distributed under the terms of the Creative Commons Attribution License, which permits unrestricted use, distribution, and reproduction in any medium, provided the original author and source are credited.

\begin{abstract}
Untargeted metabolomics has been increasingly used to evaluate metabolic alterations caused by diet, disease, or other factors in animals. The purpose of this exploratory study was to evaluate the impact of Cannabidiol (CBD) supplementation on the canine carboxyl and hydroxyl submetabolomes. Sixteen dogs (18.2 $\pm 3.4 \mathrm{~kg} \mathrm{BW}$ ) were utilized in a completely randomized design with treatments consisting of control and $4 \mathrm{mg}$ $\mathrm{CBD} / \mathrm{kg} \mathrm{BW} / \mathrm{d}$. After $21 \mathrm{~d}$ of treatment, blood was collected approximately $2 \mathrm{~h}$ after morning treat consumption. Plasma collected from samples was analyzed using CIL/LC-MS-based untargeted metabolomics to analyze carboxyl- and hydroxyl-containing metabolites. Metabolites that differed (fold change $(F C) \geq 1.2$ or $\leq 0.83$ and $F D R \leq 0.05$ ) between the two treatments were identified using a volcano plot. Biomarker analysis based on Receiver Operating Characteristic (ROC) curves was performed to identify biomarker candidates (area under ROC $\geq 0.90$ ) of the effects of CBD supplementation. Volcano plot analysis revealed that 42 carboxyl-containing metabolites and 32 hydroxyl-containing metabolites were differentially altered ( $F C \geq 1.2$ or $\leq 0.83$, FDR $\leq 0.05$ ) by CBD; these metabolites were involved in the metabolism of lipids, amino acids, carbohydrates, and more. Biomarker analysis identified 23 carboxyl-containing metabolites and 15 hydroxyl-containing metabolites as candidate biomarkers of the effects of $C B D$ (area under $R O C \geq 0.90 ; P<0.01$ ). Results of this study indicate that $4 \mathrm{mg} \mathrm{CBD} / \mathrm{kg} \mathrm{BW} / \mathrm{d}$ supplemented for 3-weeks altered the canine carboxyl and hydroxyl submetabolomes and may indicate potential mechanisms by which CBD exerts some of its effects. Future work is warranted to investigate these potential mechanisms.
\end{abstract}

Keywords: Cannabidiol; Canine; Metabolomics; Biomarkers; Metabolites

\section{Introduction}

Public perception of Cannabidiol (CBD) is overwhelmingly favorable due to the plethora of supposed health benefits, including analgesic, anxiolytic, and anti-inflammatory effects [1]. However, many of these claims have yet to be researched, and potential health and safety risks have not been thoroughly investigated. One approach to assessing health status is the use of mass spectrometry-based metabolomics, which can analyze metabolic changes in response to diet, disease, or other factors [2,3].

Two metabolomics methods are available: targeted metabolomics, which quantifies defined groups of metabolites, and untargeted metabolomics, which provides a comprehensive analysis of all measurable analytes in a sample, including unknowns. In recent years, Chemical Isotope Labeling (CIL) and Liquid ChromatographyMass Spectrometry (LC-MS)-based untargeted metabolomics has provided an opportunity to analyze metabolites based on chemical groups, including metabolites containing carboxyl groups, including fatty acids and their derivatives, and hydroxyl groups, which includes important biological compounds like hormones $[4,5]$. If there are no known metabolites of interest, untargeted metabolomics can also be used to identify specific biomarkers that can then be used in targeted metabolomics or pathway analyses in future work.

In our previous study, we applied a CIL/LC-MS-based untargeted metabolomics technique to examine differences in plasma metabolites containing amine/phenol and carbonyl chemical groups in dogs receiving CBD-containing treats compared with control [6]. However, CBD has been suggested to alter other metabolic processes, like lipid metabolism, that are not represented by those labeling methods [7]. Therefore, the objective of the current, exploratory study was to evaluate the impact of oral CBD supplementation on the canine carboxyl and hydroxyl submetabolomes using untargeted metabolomics and biomarker analysis. We hypothesized that after 3 
weeks of supplementation, CBD would alter the canine carboxyl- and hydroxyl-metabolome compared with control.

\section{Materials and Methods}

This study was approved by the Lincoln Memorial University (LMU) institutional animal care and use committee (protocol 1911RES) before the start of the study. All housing and husbandry were provided in accordance with the Animal Welfare Act, the Guide for the Care and Use of Laboratory Animals ( $8^{\text {th }}$ edition.), and all applicable LMU protocols.

\section{Subjects and diets}

Sixteen dogs ( 8 male, 8 female, 9 months to 4 years of age, $18.2 \pm 3.4$ $\mathrm{kg} \mathrm{BW}$ ) of various mixed breeds were received from a local shelter for inclusion in this study. The shelter was informed of and gave consent for the use of the dogs for research purposes before their arrival. Before beginning the experiment, each dog had a Complete Blood Count (CBC), and serum chemistry analysis (IDEXX Laboratories, Inc., Westbrook, Maine) performed, along with a physical evaluation by the attending veterinarian and a fecal examination to rule out any underlying disease that might preclude enrollment. Dogs were excluded if they demonstrated serious behavioral issues, such as aggression that would endanger research personnel, were severely emaciated, classified as a body condition score $<2$ on a 5-point scale (where 1 is emaciated and 5 is obese), or if their initial evaluations revealed an underlying disease that required more than routine treatments (i.e. heartworm positive test result).

Dogs were individually housed in $1.2 \times 1.8 \mathrm{~m}$ kennels within one of two dog wards at the LMU DeBusk Veterinary Teaching Center. They were stratified by treatment and sex and evenly distributed between the two wards. Dogs were fed Purina Pro Plan EN Gastroenteric Fiber Balance Dry Dog Food (Nestle Purina, Inc., St. Louis, MO) to meet the daily metabolizable energy requirements of neutered adult dogs at maintenance, calculated as $\left(70^{\star} \mathrm{BW}^{0.75}\right){ }^{\star} 1.6$ and split into two meals per day. Body weight and body condition score (5-point scale) were assessed weekly for the adjustment of diets. Dogs arrived from the shelter and started on the study diet more than 37 days prior to starting treatments and 58 days before collecting samples for this study.

\section{Experimental design and treatments}

These dogs were participating in a concurrent study [8] evaluating the impact of $\mathrm{CBD}$ on canine voluntary activity with treatments consisting of 0 (placebo treats; CON) and $4 \mathrm{mg} \mathrm{CBD} / \mathrm{kg}$ BW/d (CBD). Dogs were blocked by baseline activity before being stratified by age, weight, and sex and randomly assigned to treatments within each block. The CBD was a constituent of a proprietary industrial hemp extract (AgTech Scientific, Paris, KY) that was incorporated into treats and administered in the form of 1 treat twice daily, each containing half the daily dose. Both CON and CBD treats were composed of the following ingredients: chicken, chicken liver, Asian carp, catfish, and in the case of the CBD treats, industrial hemp extract. Treats were offered solely as a reward upon kennel re-entry following twice-daily exercise within $30 \mathrm{~min}$ of meals.

\section{Blood sample collection}

As previously described [6], $6 \mathrm{~mL}$ of blood was collected via cephalic catheter or jugular venipuncture after $21 \mathrm{~d}$ of treatment administration approximately 2 hours after the final treat administration. Blood samples were collected into tubes containing sodium heparin and were immediately centrifuged at $1645 \mathrm{x} g$ for $10 \mathrm{~min}$. Plasma was collected after centrifugation then stored at $-20^{\circ} \mathrm{C}(<12$ hours $)$ before long-term storage at $-80^{\circ} \mathrm{C}$.

\section{CIL/LC-MS-based untargeted metabolomics analysis}

As previously described [6], untargeted metabolomic profiling was done using a CIL/LC-MS-based technique with an Agilent 1100 LC system (Palo Alto, CA) connected to a Bruker Impact HD Quadrupole Time-Of-Flight (QTOF) MS (Billerica, MA). Detailed information regarding sample preparation, labeling, normalization, LC-UV and LC-MS setup, and metabolite quantification have been reported elsewhere. In this study, carboxyl- and hydroxyl-containing metabolites were analyzed. A total of 19 LC-MS data files were generated (3 quality control samples, $8 \mathrm{CBD}$ samples, and $8 \mathrm{CON}$ samples).

\section{Metabolite data processing}

Raw data processing on the 19 LC-MS data files was performed using ISOMS Pro 1.0 according to procedures described. Peak pairs whose mean (sample) / mean (blank) was $\leq 4.0$ were filtered out. Peak pairs with no data present in at least $80 \%$ of the samples were filtered out. The final metabolite-intensity table was generated using IsoMSQuant.

\section{Metabolite identification}

A two-tier identification approach was used to perform metabolite identification. In tier 1, peak pairs were searched against a Chemical Isotope-Labeled Metabolite Library (CIL Library) based on accurate mass and retention time. The CIL Library contains 1,213 experimental entries, including 271 carboxyls and 141 hydroxyls. In tier 2, a Linked Identity Library (LI Library) was used for the identification of the remaining peak pairs. The LI Library includes over 2000 human endogenous metabolites from 68 metabolic pathways, providing highconfidence putative identification results based on accurate mass and predicted retention time matches.

\section{Statistical analysis}

The final metabolite intensity tables for the carboxyl- and hydroxylcontaining metabolome were imported separately into Metabo Analyst 5.0 software package for statistical analysis. Before statistical analysis, the data were log-transformed, normalized by the median, and autoscaled. Median scaling eliminated unwanted inter-sample variations to make the individual samples more comparable to each other and auto-scaling made the metabolites more comparable in magnitude to each other.

Univariate (volcano plot) and multivariate analysis (Partial Least Squares Discriminant Analysis [PLS-DA] scores plot) were then generated to identify overall treatment differences across the multivariate dataset. The volcano plot was constructed by plotting the fold change (FC; $\mathrm{CBD} / \mathrm{CON}$ ) of each metabolite against its $P$-value. Metabolites with $\mathrm{FC} \geq 1.2$ or $\leq 0.83$ having a false discovery ratio (FDR) $\leq 0.05$ were considered to be differentially increased or decreased relative to $\mathrm{CON}$, respectively.

The utility of the metabolites with FC $\geq 1.2$ or $\geq 0.83$ and FDR $\leq 0.05$ to serve as potential biomarkers of the effects of CBD was tested using a Receiver Operating Characteristic (ROC) curves as calculated by the ROCCET web server using MetaboAnalyst 5.0 software package. Metabolites with an area under ROC (AUROC) $\geq 0.90$ and a $P \leq 0.05$ were considered excellent biomarkers.

\section{Results}

\section{Carboxyl-metabolome}

Within the carboxyl analysis, a total of 2,943 unique peak pairs were detected. Of those peak pairs, 29 peak pairs were positively 
identified in tier 1 (CIL Library; Supplementary Table 1) and 144 peak pairs were putatively identified with high confidence in tier 2 (LI Library; Supplementary Table 2). The PLS-DA scores plot (Figure 1A) show clear separation between $\mathrm{CON}$ and $\mathrm{CBD}$ samples, and the permutation test $(P=0.02)$ confirms the validity of the PLS-DA model (Supplementary Figure 1).

Volcano plot analysis showed that 42 metabolites were differentially altered (FC $\geq 1.2$ or $\leq 0.83$, FDR $\leq 0.05$ ) by CBD (Figure $1 \mathrm{~B}$ ). Twentyeight metabolites (Table 1 ) were differentially reduced (FC $\leq 0.83$, FDR $\leq 0.05$ ) by CBD compared to control.

Univariate analysis of the 42 carboxyl-containing metabolites positively and putatively identified that were differentially altered by CBD revealed that 23 metabolites appear to be highly predictive of the metabolomic changes between $\mathrm{CBD}$ and $\mathrm{CON}$ (AUROC $\geq 0.90 ; P<$ 0.001; Figure 2).

\section{Hydroxyl-metabolome}

Within the hydroxyl analysis, a total of 3,759 unique peak pairs were detected. Of those peak pairs, 141 peak pairs were positively identified in tier 1 (CIL Library; Supplementary Table 1) and 65 peak pairs were putatively identified with high confidence in tier 2 (LI Library; Supplementary Table 2). The PLS-DA scores plot (Figure $3 \mathrm{~A})$ show a clear separation between $\mathrm{CON}$ and CBD samples, and the permutation test $(P<0.01)$ confirms the validity of the PLS-DA model (Supplementary Figure 2).

Volcano plot analysis showed that 32 metabolites were differentially altered $(\mathrm{FC} \geq 1.2$ or $\leq 0.83$, FDR $\leq 0.05)$ by CBD (Figure $3 \mathrm{~B}$; Table 2 ). Fourteen metabolites were differentially increased (FC $\geq 1.5$, FDR $\leq$ $0.05)$ by CBD. Eighteen metabolites were differentially reduced (FC $\leq$ $0.83, \mathrm{FDR} \leq 0.05$ ) by CBD compared with control.

Univariate analysis of the 35 hydroxyl-containing metabolites positively and putatively identified that were differentially increased or decreased by CBD revealed that 15 metabolites appear to be highly predictive of the metabolomic changes between $\mathrm{CBD}$ and $\mathrm{CON}$ (AUROC $\geq 0.90 ; P<0.001$; Figure 4 ).

\section{Discussion}

\section{Lipid metabolism}

Increased concentrations of $17 a, 20 a$-dihydroxypregn-4-en-3-one, ethyl malonate, 3-hydroxybutyric acid, an isomer of 3-hydroxybutyric acid, 3-hydroxypropionic acid, an isomer of hydroxypropionic acid, and butyric acid may indicate an alteration of lipid metabolism with CBD supplementation. Primarily known as a steroid hormone elevated in late pregnancy in mammals, 17a, 20a-dihydroxypregn-4-en-3-one is also thought to play a role in progesterone homeostasis in nonpregnant animals $[9,10]$. Ethyl malonate is a branched fatty acid associated with several fatty acid metabolism disorders in humans, including short-chain acyl-CoA dehydrogenase deficiency and ethylmalonic encephalopathy $[11,12]$, and has also been identified as a potential biomarker for the detection of human breast cancer [13]. 3-hydroxybutyric acid is a ketone body and a typical intermediate in the partial breakdown of branched-chain amino acids like valine in muscles [14]. 3-Hydroxypropionic acid is an intermediate in beta-alanine, propanoate, and uracil metabolism $[15,16]$. Butyric acid is an intermediate in butanoate metabolism, which is often formed as a result of bacterial fermentation in the gut $[17,18]$. Many of these changes could also be impacted by changes to the microbiome which has not been previously linked to $\mathrm{CBD}$ supplementation.
Additionally, decreased concentrations of propane-1,3diol, glycerol, ethanolamine, an isomer of 9-oxononanoic acid, 9,10-12,13-diepoxyoctadecanoate, allotetrahydrodeoxycorticosterone 3-O-glucuronide, cortolone, and cortisol 21-O-sulfate may also suggest that $\mathrm{CBD}$ altered lipid metabolism. Propane-1,3-diol is a product of glycerol fermentation in bacteria but is not known as a mammalian metabolite [19]. Glycerol is the backbone of glycerides and plays an essential role in lipid metabolism, carbohydrate metabolism, and other metabolic processes [20]. Blood glycerol is also a biomarker for liver disease, hyperglycemia, and type II diabetes $[21,22]$. Additionally, glycerol is involved in endocannabinoid signaling as a product of the endocannabinoid 2-arachidonylglycerol hydrolysis [23]. Ethanolamine, an amino alcohol, is an intermediate in glycerophospholipid metabolism. It is also involved in retrograde endocannabinoid signaling as a product of arachidonoyl ethanolamide degradation by fatty acid amide hydrolase [24]. Collectively, these results may suggest $\mathrm{CBD}$ inhibited arachidonoyl ethanolamide degradation, which supports previous work showing inhibition of cellular uptake and breakdown of arachidonoyl ethanolamide by CBD via inhibition of fatty acid binding proteins [25-27].

9-Oxononanoic acid is a medium-chain fatty acid product of alphalinoleic acid oxidation in plants [28]. Early studies showed elevated lipid peroxides and reduced lipogenesis in rat livers after oral administration of 9-oxononanoic acid [29,30]; more recent work indicates this is a result of arachidonic cascade induction by 9-oxononanoic acid through its activation of phospholipase $A_{2}$ [31]. If the decrease in 9-oxononanoic acid is a result of CBD supplementation, this may be one potential mechanism contributing to the suspected antiinflammatory effects of CBD. Jasmonic acid is a plant stress hormone synthesized from linolenic acid that serves as a natural pesticide and promotes plant growth [32]. Additionally, jasmonic acid has been suggested to exhibit anti-cancer and anti-inflammatory properties in vitro [33]. 9,10-12,13- Diepoxyoctadecanoate is an intermediate in the conversion of linoleic acid into tetrahydrofurandiols [34].

Allotetrahydrodeoxycorticosterone 3-O-glucuronide is an endogenous neurosteroid that acts as a potent positive allosteric modulator of the $\mathrm{GABA}_{\mathrm{A}}$ receptor and exhibits sedative, anxiolytic, and anticonvulsant effects $[35,36]$. Cortolone and cortisol 21-O-sulfate are metabolites in steroid hormone catabolism [37]. Cortolone is often linked to a glucuronide to help in its excretion [38,39], and cortisol 21-O-sulfate may be used as a biomarker for Cushing's syndrome [37].

Altogether, these results support an effect of $\mathrm{CBD}$ on lipid metabolism and may indicate potential mechanisms for its suspected anti-inflammatory effect. However, as the animals on this study were healthy, it is unclear how this would translate to a diseased or aged population in which these metabolomic changes might be more influential, such as animals with Cushing's disease. Further studies are needed in unhealthy or diseased populations of dogs to determine the potential physiological applications of these potential effects of CBD.

\section{Carbohydrate metabolism}

Increased concentrations of lactic acid, acetic acid, D-glycerate, D-tagatose, D-galactosamine, L-rhamnono-1,4-lactone, L-rhamnofuranose, L-fuculose, 6-deoxy-L-galactose, and 5-deoxy-Dglucuronate may indicate that $\mathrm{CBD}$ altered carbohydrate metabolism. Lactic acid is generated from pyruvate during anaerobic conditions in the muscle and red blood cells. The Cori cycle, or lactic acid cycle, removes lactate from tissues and transports lactate via blood to the liver where it can be converted back into glucose [40,41]. As both this cycle and lactic acid play vital roles in glucose homeostasis, the increase 
Table 1: Identified carboxyl-containing metabolites affected by cannabidiol (CBD) compared to control (CON). Metabolites with a fold change (FC) $\geq 1.2$ relative to CON and a false discovery ratio (FDR) $\leq 0.05$ considered increased in the CBD compared to CON. Metabolites with a FC $\leq 0.83$ and an FDR $\leq$ 0.05 considered reduced in CBD compared to CON.

\begin{tabular}{|c|c|c|c|c|}
\hline Metabolite & Normalized $\mathbf{R T}^{1}$ & FC & FDR & Identification Level $^{2}$ \\
\hline 2,5-Dioxopentanoate & 741.4 & 3.70 & 0.047 & Tier 2 \\
\hline Isomer of Hydroxypropionic Acid & 380.9 & 2.56 & $<.001$ & Tier 1 \\
\hline 4-Coumarate & 802.6 & 2.42 & 0.043 & Tier 2 \\
\hline Isomer of D-Glycerate & 255.0 & 2.31 & 0.009 & Tier 2 \\
\hline Acetic Acid & 560.5 & 2.21 & $<.001$ & Tier 1 \\
\hline D-Glycerate & 299.4 & 2.14 & 0.007 & Tier 2 \\
\hline Isomer of Glycolate & 282.4 & 2.09 & 0.008 & Tier 2 \\
\hline Isomer of Threonate & 272.2 & 2.09 & 0.088 & Tier 2 \\
\hline 5-Deoxy-D-Glucuronate & 361.4 & 2.04 & 0.002 & Tier 2 \\
\hline Citramalic Acid & 449.1 & 2.04 & 0.001 & Tier 1 \\
\hline 3,4-Dihydroxymandelic Acid & 493.9 & 2.04 & $<.001$ & Tier 1 \\
\hline Glycolate & 299.4 & 2.03 & 0.046 & Tier 2 \\
\hline 3-Oxopropanoate & 361.4 & 1.95 & 0.002 & Tier 2 \\
\hline Ethyl Malonate & 739.7 & 1.89 & $<.001$ & Tier 1 \\
\hline Isomer of 3-Oxopropanoate & 332.4 & 1.88 & 0.007 & Tier 2 \\
\hline Isomer of 3-Hydroxybutyric Acid & 474.9 & 1.86 & 0.005 & Tier 1 \\
\hline 2-Hydroxy-3-Oxopropanoate & 389.2 & 1.81 & 0.080 & Tier 2 \\
\hline Hydroxyisobutyric Acid & 548.8 & 1.60 & 0.012 & Tier 1 \\
\hline Isovaleric Acid & 1039.8 & 1.60 & 0.030 & Tier 1 \\
\hline Butyric Acid & 870.3 & 1.58 & 0.009 & Tier 1 \\
\hline Glyoxylate & 528.3 & 1.55 & 0.083 & Tier 2 \\
\hline Nicotinate & 574.9 & 1.54 & 0.007 & Tier 2 \\
\hline 3-Hydroxybutyric Acid & 501.4 & 1.43 & 0.047 & Tier 1 \\
\hline S-5-Amino-3-Oxohexanoic Acid & 596.0 & 1.38 & 0.025 & Tier 2 \\
\hline Hydroxypropionic Acid & 424.8 & 1.38 & 0.015 & Tier 1 \\
\hline Lactic Acid & 452.2 & 1.37 & 0.012 & Tier 1 \\
\hline Isomer of Lactic Acid & 471.2 & 1.36 & 0.009 & Tier 1 \\
\hline Isovanillic Acid & 739.5 & 1.33 & 0.009 & Tier 1 \\
\hline 4-Oxoproline & 798.3 & 0.80 & 0.017 & Tier 2 \\
\hline 2-Aminomuconate Semialdehyde & 799.6 & 0.79 & 0.032 & Tier 2 \\
\hline Isomer of 9-Oxononanoic Acid & 1048.9 & 0.74 & 0.093 & Tier 2 \\
\hline L-1-Pyrroline-3-Hydroxy-5-Carboxylate & 372.5 & 0.74 & 0.012 & Tier 2 \\
\hline S-4-Amino-5-oxopentanoate & 439.3 & 0.70 & 0.007 & Tier 2 \\
\hline Isomer of 1-Aminocyclopropane-1-Carboxylate & 272.2 & 0.67 & 0.013 & Tier 2 \\
\hline L-Allothreonine & 439.3 & 0.64 & 0.007 & Tier 2 \\
\hline Arginine & 279.8 & 0.60 & 0.012 & Tier 2 \\
\hline Isomer of Aspartate & 574.6 & 0.58 & 0.098 & Tier 2 \\
\hline Isomer of Jasmonic Acid & 1525.6 & 0.55 & 0.030 & Tier 2 \\
\hline 2-Oxo-4-Phenylbutyric Acid & 1150.3 & 0.53 & 0.007 & Tier 2 \\
\hline 9,10-12,13-Diepoxyoctadecanoate & 1333.8 & 0.50 & 0.009 & Tier 2 \\
\hline Jasmonic Acid & 1437.0 & 0.44 & $<.001$ & Tier 2 \\
\hline Isomer of 1-Pyrroline-2-Carboxylate & 1160.7 & 0.15 & 0.009 & Tier 2 \\
\hline
\end{tabular}

${ }^{1}$ Normalized RT (retention time) shows the corrected retention time of the peak pair with Universal RT Calibrant data.

${ }^{2}$ Tier 1 indicates positive metabolite identification within the chemical isotope labeling (CIL) metabolite library whereas Tier 2 indicates high confidence putative identification within the linked identity (LI) library. 
Table 2: Identified hydroxyl-containing metabolites affected by cannabidiol (CBD) compared to control (CON). Metabolites with a fold change (FC) $\geq 1.2$ relative to CON and a false discovery ratio (FDR) $\leq 0.05$ considered increased in the CBD compared to CON. Metabolites with a FC $\leq 0.83$ and an FDR $\leq$ 0.05 considered reduced in CBD compared to CON.

\begin{tabular}{|c|c|c|c|c|}
\hline Metabolite & Normalized $\mathbf{R T}^{1}$ & FC & FDR & Identification Level $^{2}$ \\
\hline 17alpha,20alpha-Dihydroxypregn-4-en-3-one & 1618.8 & 6.19 & 0.001 & Tier 2 \\
\hline D-Tagatose & 235.4 & 2.35 & 0.001 & Tier 2 \\
\hline 3,4-Dihydroxyphenylpropanoate & 678.7 & 2.09 & 0.011 & Tier 2 \\
\hline L-Rhamnono-1,4-Lactone & 280.5 & 1.91 & 0.001 & Tier 2 \\
\hline L-Rhamnofuranose & 198.0 & 1.67 & 0.008 & Tier 2 \\
\hline Isomer of L-Rhamnofuranose & 735.3 & 1.66 & 0.008 & Tier 2 \\
\hline 6-Deoxy-L-Galactose & 649.6 & 1.66 & 0.008 & Tier 2 \\
\hline D-Galactosamine & 162.8 & 1.48 & 0.008 & Tier 2 \\
\hline Sepiapterin & 240.1 & 1.48 & 0.014 & Tier 2 \\
\hline Isomer of Sepiapterin & 182.5 & 1.46 & 0.028 & Tier 2 \\
\hline L-Fuculose & 128.6 & 1.43 & 0.001 & Tier 2 \\
\hline Isomer of Deoxyadenosine & 261.6 & 1.42 & 0.019 & Tier 2 \\
\hline Deoxyadenosine & 243.3 & 1.40 & 0.035 & Tier 2 \\
\hline 3-Hydroxy-L-Proline & 211.6 & 1.23 & 0.016 & Tier 2 \\
\hline N-Acetyl-trans-3-Hydroxy-L-Proline & 602.8 & 0.82 & 0.080 & Tier 2 \\
\hline Ethanolamine & 398.0 & 0.82 & 0.045 & Tier 2 \\
\hline 2,3-Dihydroxyindole & 578.9 & 0.81 & 0.044 & Tier 2 \\
\hline N2'-Acetyl-3'-Hydroxykynurenamine & 425.7 & 0.79 & 0.087 & Tier 2 \\
\hline Cyanate & 469.9 & 0.79 & 0.014 & Tier 2 \\
\hline Isomer of Cyanate & 505.6 & 0.77 & 0.008 & Tier 2 \\
\hline Glycerol & 447.9 & 0.76 & 0.051 & Tier 2 \\
\hline Alpha-Ribazole & 894.1 & 0.74 & 0.014 & Tier 2 \\
\hline Isomer of 3-Phenoxybenzyl Alcohol & 538.1 & 0.73 & 0.008 & Tier 1 \\
\hline Dihydroshikonofuran & 1341.0 & 0.73 & 0.018 & Tier 2 \\
\hline Glycolaldehyde & 613.0 & 0.71 & 0.018 & Tier 2 \\
\hline N-Acetyl-2-Carboxy-2,3-Dihydro-5,6-Dihydroxyindole & 404.7 & 0.69 & 0.014 & Tier 2 \\
\hline Allotetrahydrodeoxycorticosterone 3-O-Glucuronide & 1167.4 & 0.69 & 0.027 & Tier 2 \\
\hline Phenethyl Alcohol & 653.4 & 0.63 & 0.002 & Tier 1 \\
\hline Propane-1,3-Diol & 945.6 & 0.56 & 0.001 & Tier 2 \\
\hline Cortolone & 1011.9 & 0.46 & 0.092 & Tier 2 \\
\hline Arabitol & 238.7 & 0.42 & 0.001 & Tier 2 \\
\hline Cortisol 21-O-Sulfate & 875.1 & 0.40 & 0.088 & Tier 2 \\
\hline
\end{tabular}

${ }^{1}$ Normalized RT (retention time) shows the corrected retention time of the peak pair with Universal RT Calibrant data.

${ }^{2}$ Tier 1 indicates positive metabolite identification within the chemical isotope labeling (CIL) metabolite library whereas Tier 2 indicates high confidence putative identification within the linked identity (LI) library.

in plasma lactic acid indicates alteration of glucose metabolism by CBD. Similarly, acetic acid plays a role as an intermediate in several essential metabolic pathways, including glycolysis, pyruvate metabolism, and glyoxylate metabolism via acetyl-CoA. These results support our previous work investigating the impact of CBD supplementation on amine/phenol- and carbonyl-containing metabolites, in which glucose and several other metabolites associated with energy metabolism were altered [6], and highlight the importance of continued work on the potential anti-obesity and antidiabetic effects of CBD.
D-glycerate is an intermediate in several metabolic pathways. Regarding carbohydrate metabolism, D-glycerate is an intermediate in the conversion of other D-sugars and glyoxylate to 2-phosphoglycerate, which can then feed into glycolysis [42,43]. D-tagatose is a monosaccharide often found in fruit or dairy products that are commonly used as an artificial sweetener due to its low glycemic index [44]. It can also be produced as an intermediate in the catabolism of $\mathrm{D}$-sugars like $\mathrm{D}$-galactose before entry into the pentose phosphate pathway [45]. D-galactosamine is an amino sugar derived from galactose that is a constituent of glycoprotein 
1A)

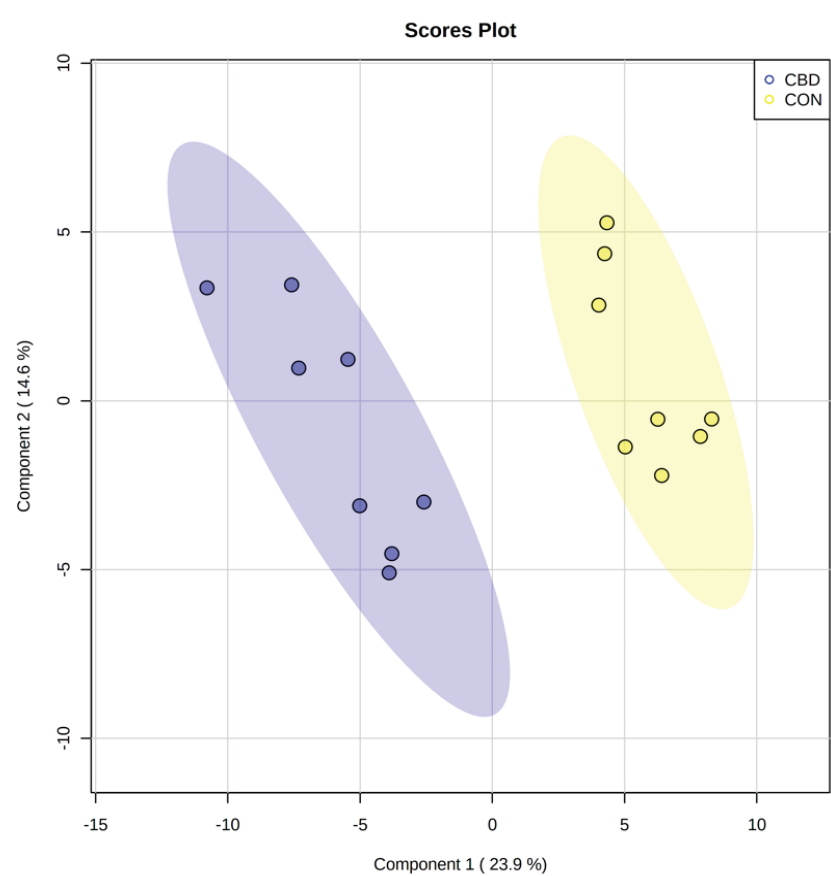

1B)

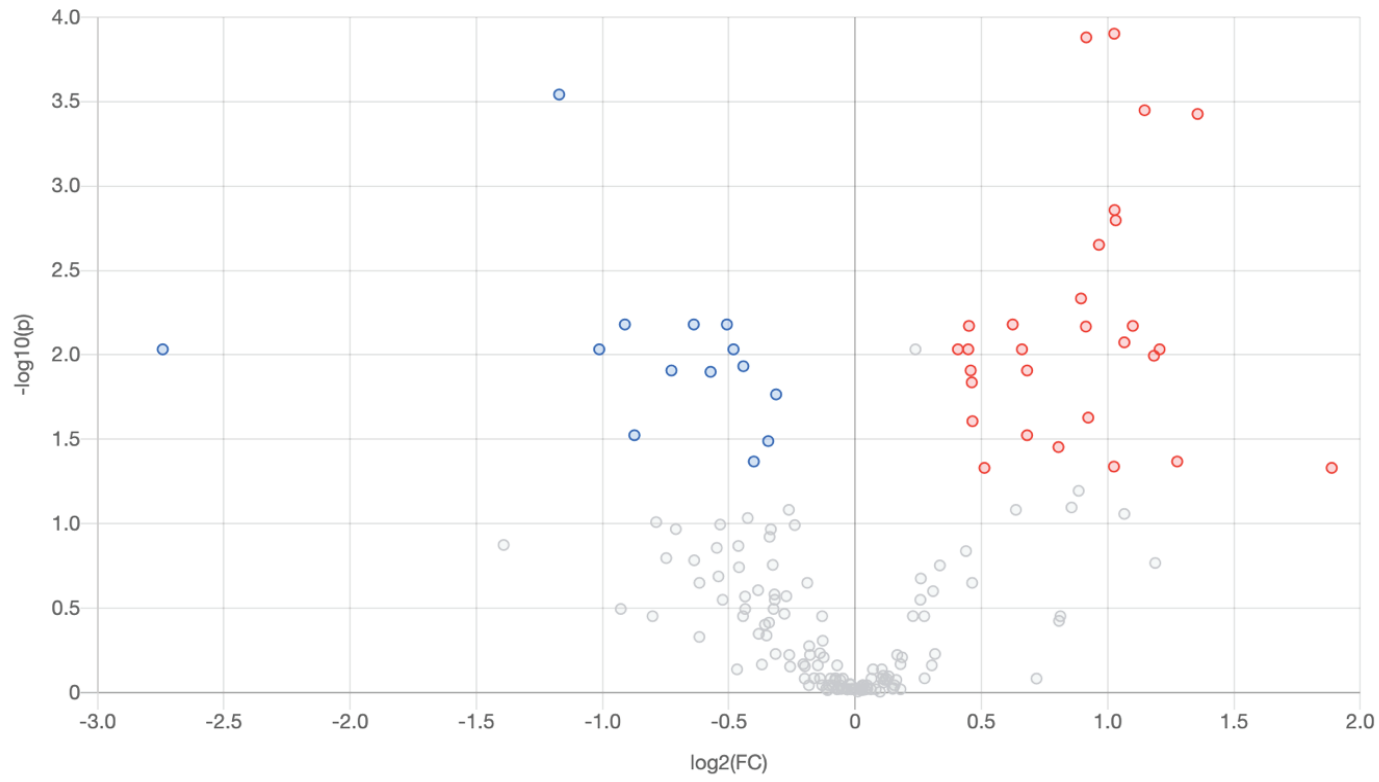

Figure 1: A. Partial least squares discriminant analysis (PLS-DA) scores plot and B. volcano plot showing the differential carboxyl-containing metabolites. Fold change $(F C) \geq 1.2$ (in red) or $\leq 0.83$ (in blue) with false discovery ratio (FDR) $\leq 0.05$ are differentially increased or reduced by cannabidiol (CBD) relative to control (CON).

hormones like luteinizing hormone and follicle-stimulating hormone [46,47].

L-rhamnono-1,4-lactone, L-rhamnofuranose, L-fuculose, and 6-deoxy-L-galactose (i.e. fucose) are intermediates in fructose and mannose metabolism [48-50]. Fucose, in particular, is a common $\mathrm{N}$-linked glycan that has been shown to play an important role in mammalian health, with disruption of fucosylated glycan expression implicated in a number of disease mechanisms [48,51].

5-deoxy-D-glucuronate is an intermediate in the catabolism of myoinositol in bacterial species like Bacillus subtilis, a common species found in the gastrointestinal tract of humans and ruminants [52,53]. While not known to be produced in canine metabolic pathways, production of 5-deoxy-D-glucuronate in the gastrointestinal tract by micro flora may have been absorbed into the body. The increase in this metabolite may suggest CBD supplementation affected gastrointestinal micro flora populations.

Additionally, decreased concentrations of arabitol may suggest an impact of CBD on the microbiome. Arabitiol is a sugar alcohol commonly produced by several yeast species [54]. Elevated levels of arabitol in urine have been suggested as a biomarker of fungal 

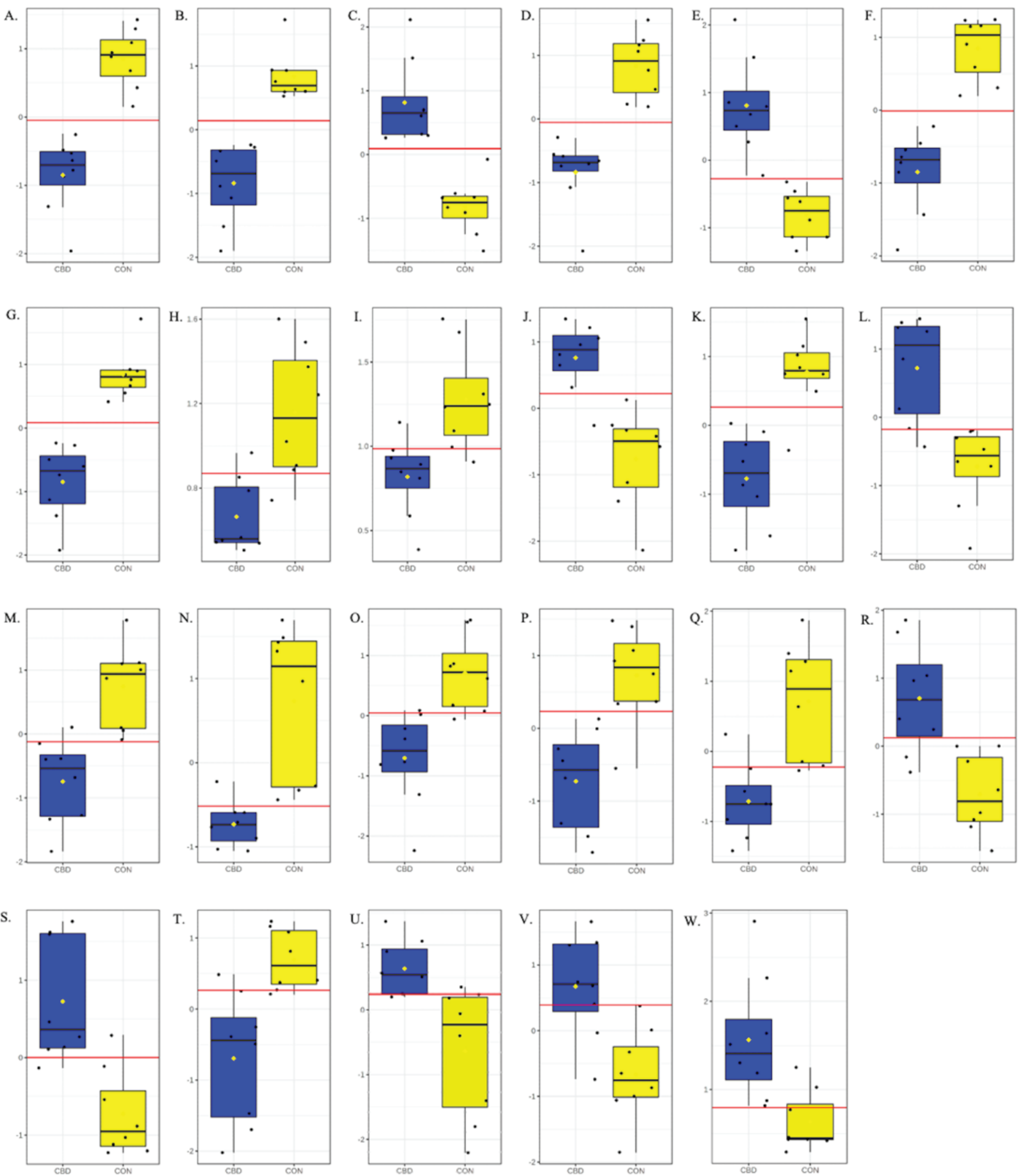

Figure 2: Biomarker analysis of carboxyl metabolites for $C B D(C B D)$ and control $(C O N)$ treatments identified $A$. jasmonic acid $(A U R O C=1.00$; $\mathrm{P}<0.001)$; B. (S)-4-amino-5-oxopentanoate $(A U R O C=1.00 ; \mathrm{P}<0.001)$; C. 3,4-dihydroxymandelic acid $(\mathrm{AUROC}=1.00 ; \mathrm{P}<0.001)$; $\mathrm{D}$. isomer of 1-aminocyclopropane-1-carboxylate (AUROC = 1.00; P < 0.001); $\mathrm{E}$. isomer of hydroxypropionic acid (AUROC = 1.00; P<0.001); F. L-1-pyrroline-3hydroxy-5-carboxylate (AUROC = 1.00; $\mathrm{P}<0.001)$; G. L-allothreonine (AUROC = 1.00; $\mathrm{P}<0.001) ;$ H. 2-oxo-4-phenylbutyric acid $(A U R O C=0.98 ; \mathrm{P}<$ 0.001); I. Arginine (AUROC $=0.98 ; P<0.001)$; J. citramalic acid (AUROC $=0.97 ; P<0.001) ;$ K. 4-oxoproline $(A U R O C=0.95 ; P<0.001)$; L. 5-deoxy-Dglucuronate (AUROC $=0.95 ; \mathrm{P}<0.001) ; \mathbf{M} .9,10-12,13$-diepoxyoctadecanoate $(A U R O C=0.95 ; \mathrm{P}<0.001)$; $\mathbf{N}$. isomer of 1-pyrroline-2-carboxylate (AUROC $=0.95 ; \mathrm{P}<0.001) ; \mathrm{O}$. isomer of aspartate $(A \cup R O C=0.95 ; \mathrm{P}<0.001) ; \mathrm{P} .2$-aminomuconate semialdehyde $(\mathrm{AUROC}=0.94 ; \mathrm{P}<0.001) ; \mathrm{Q}$. isomer of 9-oxononanoic acid (AUROC $=0.94 ; \mathrm{P}<0.001)$; $R$. acetic acid $(A U R O C=0.92 ; \mathrm{P}<0.001)$; . ethyl malonate $(A U R O C=0.92 ; P<0.001)$; T. isomer of jasmonic acid (AUROC $=0.92 ; \mathrm{P}<0.001)$; U. 3-oxopropanoate $(A U R O C=0.91 ; \mathrm{P}<0.001)$; V. D-glycerate $(A U R O C=0.91 ; P<0.001)$; and $\mathbf{W}$. isomer of $\mathrm{D}$-glycerate $(A \cup R O C=0.91 ; P<0.001)$ as candidate biomarkers. 
3A)

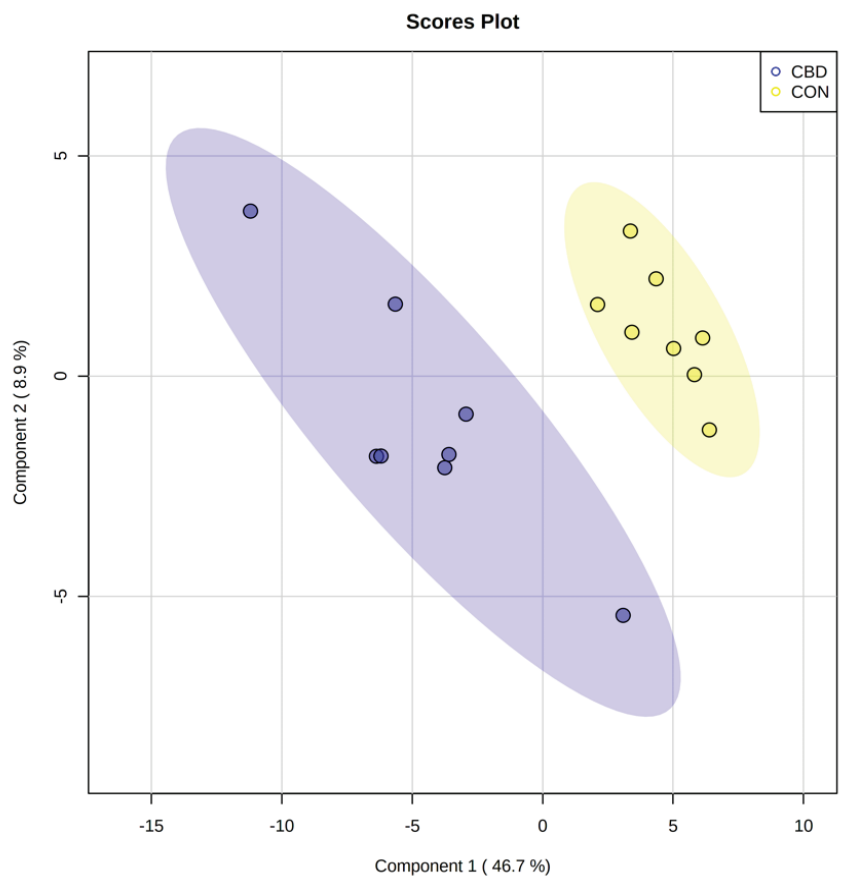

3B)

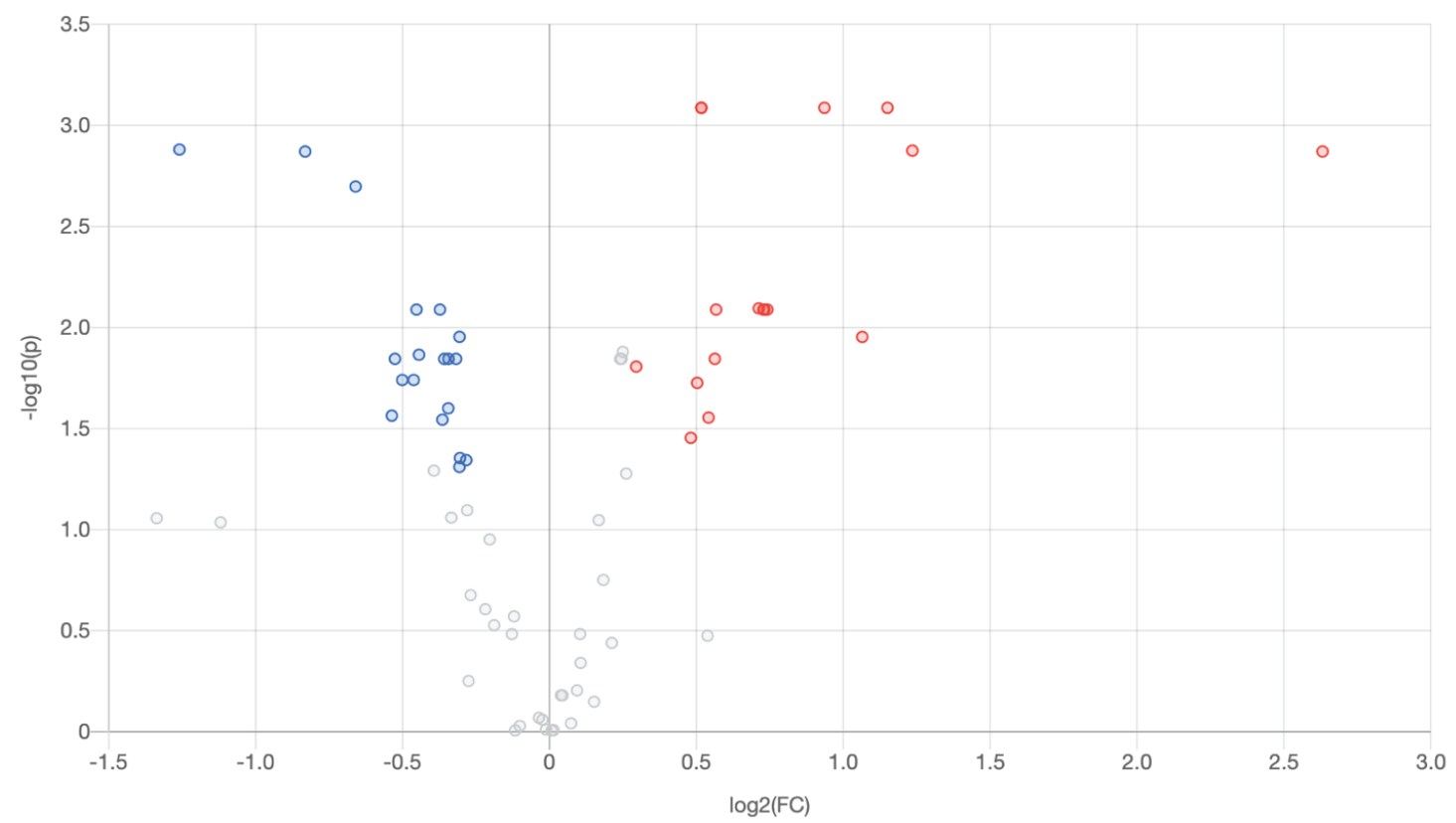

Figure 3: A. Partial least squares discriminant analysis (PLS-DA) scores plot and B. volcano plot showing the differential hydroxyl-containing metabolites. Fold change $(F C) \geq 1.2$ (in red) or $\leq 0.83$ (in blue) with false discovery ratio (FDR) $\leq 0.05$ are differentially increased or reduced by cannabidiol (CBD) relative to control (CON).

infections [55], and elevated plasma arabitol has been linked to other diseases like congenital liver cirrhosis and acute mountain sickness in humans [56,57]. While CBD is believed to be beneficial in an assortment of gastrointestinal conditions [58], little work has been done regarding its influence on microbial populations in the canine gastrointestinal tract, highlighting a potential avenue for future investigation of CBD supplementation.

\section{Amino acid metabolism}

Increased concentrations of 2,5-dioxopentanoate, 3-hydroxy-
L-proline, D-glycerate, isomer of D-glycerate, glycolate, isomer of glycolate, glyoxylate, 2-hydroxy-3-oxopropanoate, glycolaldehyde 3,4-dihydroxymandelic acid, hydroxyisobutyric acid, isovaleric acid, butyric acid, S-5-amino-3-oxohexanoic acid, 3-hydroxypropionic acid, and isomer of hydroxypropionic acid all indicate that CBD altered amino acid metabolism.

2,5-dioxopentanoate is an intermediate in the catabolism of 4-hydroxyproline in bacteria [59]. It can be converted into 2-oxoglutarate, by which it can enter the citric acid cycle through 

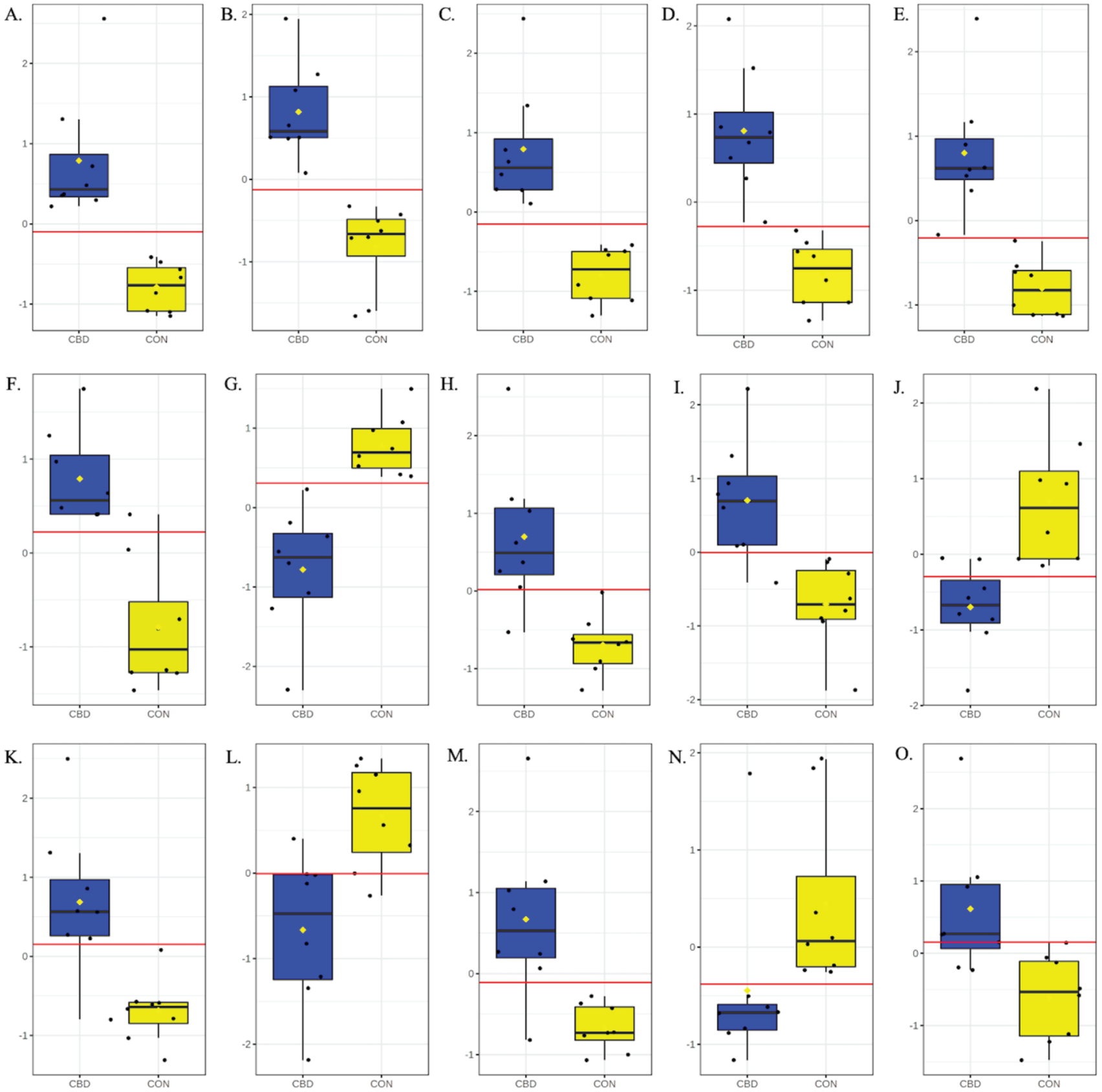

Figure 4: Biomarker analysis of hydroxyl metabolites for CBD (CBD) and control $(C O N)$ treatments identified $A$. $L-f u c u l o s e(A U R O C=1.00 ; P<$ $0.001)$; B. glyceraldehyde $(A U R O C=1.00 ; P<0.001)$; C. L-rhamnofuranose (AUROC = 1.00; $P<0.001)$; D. L-rhamnono-1,4-lactone $(A U R O C=1.00 ; P$ $<0.001)$; E. Arabitol (AUROC = 1.00; P < 0.001); F. D-tagatose (AUROC = 0.98; P<0.001); G. propane-1,3-diol (AUROC =0.98; P<0.001); H. 6-deoxyL-galactose (AUROC = 0.97; $P=0.002) ; \mathrm{I}$. D-galactosamine (AUROC = 0.95; $P=0.001)$; J. phenethyl alcohol $(A U R O C=0.94 ; P=0.002) ; K$. isomer of L-rhamnofuranose (AUROC = 0.92; P = 0.001); L. 3-phenoxybenzyl alcohol (AUROC =0.91; P = 0.003); M. 3,4-dihydroxyphenylpropanoate (AUROC $=0.91 ; \mathrm{P}=0.003) ; \mathbf{N}$. cortolone $(\mathrm{AUROC}=0.91 ; \mathrm{P}=0.073)$; and $\mathbf{O}$. isomer of deoxyadenosine $(\mathrm{AUROC}=0.91 ; \mathrm{P}=0.008)$ as candidate biomarkers.

conversion into succinyl-CoA in several prokaryotic organisms, including several species of Pseudomonas, Escherichia coli, and Haloferax volcanii [60-62]. Isovaleric acid is a branched-chain fatty acid intermediate in leucine catabolism [63], and S-5-Amino-3oxohexanoic acid is an intermediate in lysine degradation [64,65].

3-Hydroxy-L-proline is an intermediate in arginine and proline metabolism [66]. D-glycerate is an essential intermediate in the catabolism of glycine, serine, and threonine in plants. Once formed, D-glycerate can then feed into either glyoxylate metabolism via hydroxypyruvate or be converted into 3-phospho-D-glycerate and fed into glycolysis $[67,68]$. In humans with a glycerate kinase mutation, D-glycerate levels are elevated to the point of acidemia which, if left untreated, can lead to progressive neurological impairment, hypotonia, seizures, failure to thrive, and metabolic acidosis $[69,70]$. 
In plants, glycolate is an intermediate in glyoxylate metabolism, converting hydroxypyruvate into glyoxylate for further metabolism in the glyoxylate cycle [71]. In mammalian systems, glyoxylate is produced either through oxidation of glycolate in peroxisomes or the catabolism of hydroxyproline before being converted into glycine via alanine-glyoxylate aminotransferases present in peroxisomes [72,73]. The glyoxylate cycle, a pathway that converts fatty acids into glucose once believed to be absent in mammalian systems, may be present in the liver [74,75]. Genetic defects in glyoxylate metabolic enzymes have been attributed to metabolic diseases like primary hyperoxalurias and insulin resistance, though this has not been investigated in canine models $[73,75]$. Additionally, plasma glyoxylate has been indicated as an early marker for type II diabetes development in humans [76,77]. 2-Hydroxy-3-oxopropanoate and glycolaldehyde are also additional intermediates in glyoxylate metabolism $[78,79]$.

Glycolaldehyde is also a precursor for pyridoxine synthesis and is an intermediate in folate synthesis in bacteria [80].3,4-Dihydroxymandelic acid is a metabolite of norepinephrine with potent antioxidant activity [81]. Hydroxyisobutyric acid is an intermediate in valine degradation that in humans is used as a biomarker of 3-hydroxyisobutyric aciduria and methylmalonic semialdehyde dehydrogenase deficiency, rare metabolic diseases [82]. Alpha-hydroxyisobutyric acid was shown to be upregulated in a small group of dogs with diabetes compared to healthy controls [83], though it was not identified as a potential CBD biomarker in this study.

Decreased concentrations of 4-oxoproline, L-1-pyrroline-3hydroxy-5-carboxylate, isomer of 1-pyrroline-2-carboxylate, $\mathrm{N}$-acetyltrans-3-Hydroxy-L-proline, arginine, 2-aminomuconate semialdehyde, isomer of 1-aminocyclopropane-1-carboxylate, L-allothreonine, isomer of aspartate, 2-oxo-4-phenylbutyric acid, 2,3-dihydroxyindole, $\mathrm{N} 2$ '-acetyl-3'-hydroxykynurenamine, cyanate, isomer of cyanate, and $\mathrm{N}$-acetyl-2-Carboxy-2,3-dihydro-5,6-dihydroxyindole may also point to an alteration of amino acid metabolism by CBD. 4-oxoproline, L-1pyrroline-3-hydroxy-5-carboxylate, and 1-pyrroline-2-carboxylate are intermediates in arginine and proline metabolism [84-86]. N-Acetyltrans-3-hydroxy-L-proline (i.e. oxaceprol) is a derivative of L-proline that is an established anti-inflammatory drug used in the treatment of osteoarthritis [87]. Combined with the decrease in arginine, these results suggest an impact of $\mathrm{CBD}$ on arginine and proline metabolism; however, since the relative concentration of proline was unaffected by treatment, the biological significance is unclear.

2-Aminomuconate semialdehyde, 2,3-dihydroxyindole, and N2'acetyl-3'-hydroxykynurenamide are intermediates in tryptophan metabolism. 2-Aminomuconate is part of the kynurenine pathway, a metabolic pathway used for NAD biosynthesis $[88,89]$. Kynurenine pathway metabolites like 2-aminomuconate are thought to help regulate processes like immune cell response, neuronal excitability, and host-microbiome signaling [90]. N2'-Acetyl-3'Hydroxykynurenamine is an acetylated intermediate in tryptophan metabolism [91]. 2-3-Dihydroxyindole is an intermediate in an indole degradation pathway present in several bacterial species but is not known as a mammalian metabolite [92].

2-Oxo-4-phenylbutyric acid is an intermediate in phenylalanine, tyrosine, and tryptophan metabolism that, in microbial species, is a precursor to homophenylalanine [93]; however, this pathway is not known to be present in mammalian species. N-Acetyl-2-Carboxy2,3-dihydro-5,6-dihydroxyindole (i.e. leucodopachrome) is an intermediate in tyrosine metabolism and in the betalain melanogenesis pathway $[94,95]$. Cyanate is an intermediate in nitrogen metabolism that can be produced spontaneously from urea. It has been suggested to improve insulin sensitivity and potentially exert antioxidative effects [96].

\section{Vitamin and nucleotide metabolism}

Increased concentrations of an isomer of threonate, 2-hydroxy3-oxopropanoate, nicotinate, 3-oxopropanoate, an isomer of 3-oxopropanoate, deoxyadenosine, and an isomer of deoxyadenosine may suggest an alteration of vitamin and nucleotide metabolism by CBD. Threonate is an intermediate in ascorbate metabolism that is thought to play a role in bone mineralization, prevent androgendriven balding, and improve learning and memory when combined with magnesium [97-99]. Nicotinate, or vitamin B3, is required for the formation of coenzymes NAD and NADP. While it can be synthesized from tryptophan in humans and dogs, the process is inefficient and is thus considered an essential nutrient $[100,101]$.

3-Oxopropanoate is an intermediate in uracil and beta-alanine metabolism that can then be converted into malonate and enter fatty acid biosynthetic pathways [102]. Deoxyadenosine is a derivative of adenosine and an intermediate in purine metabolism. In the absence of adenosine deaminase in humans, deoxyadenosine will accumulate in and kill $\mathrm{T}$ lymphocytes, leading to the development of adenosine deaminase severe combined immunodeficiency disease [103].

Decreased concentrations of alpha-ribazole also indicate CBD altered vitamin metabolism. alpha-Ribazole is an intermediate in vitamin B12 synthesis in plants and bacterial species that can be used as a marker for vitamin B12 content in foodstuffs $[104,105]$.

\section{Additional metabolites}

Increased relative concentrations of 4-coumarate, 3,4-dihydroxyhpenylpropanoate, isovanillic acid, citramalic acid, sepiapterin, and an isomer of sepiapterin may further suggest an alteration of metabolism by CBD supplementation. 4-Coumarate, or 4-hydroxycinnamate, is a hydroxycinnamic acid derivative and an intermediate in several plant metabolic pathways, including phenylpropanoid biosynthesis, isoquinoline alkaloid biosynthesis, tyrosine metabolism, and more [106,107]. 3,4-Dihydroxyphenylpropanoate, or dihydrocaffeic acid, is an intermediate in tyrosine metabolism, specifically the conversion of L-DOPA to rosmarinate, and is suspected to possess antioxidant and anti-inflammatory properties $[108,109]$.

Isovanillic acid is a metabolite of isovanillin with suspected antibacterial properties $[110,111]$. While not known as a mammalian metabolite, it can be found in plasma after flavonoid consumption [112]. Citramalic acid is most commonly produced by yeast or anaerobic bacteria as an intermediate in C5-branched dibasic acid metabolism [113]. As it is not a mammalian metabolite, citramalic acid is most commonly found as a urine metabolite in cases of yeast or bacterial overgrowth [114]. Sepiapterin is an intermediate in folate biosynthesis that in mammals can be metabolized into tetrahydrobiopterin, which is an essential cofactor in mammals for the metabolism of aromatic amino acids and biosynthesis of neurotransmitters [115,116]. It has been suggested as a treatment for atherosclerosis and tetrahydropterin deficiencies in humans [115-118].

Decreased concentrations of L-glutamate-1-semialdehyde, 1-aminocyclopropane-1-carboxylate, L-allothreonine, an isomer of 3-phenoxybenzyl alcohol, dihydroshikonofuran, and phenethyl alcohol also indicate an effect of CBD on metabolism.L-Glutamate1-semialdehyde, 1-aminocyclopropane-1-carboxylate, and L-allothreonine are plant metabolites. L-Glutamate-1-semialdehyde is produced as an intermediate in porphyrin and chlorophyll synthesis 
[119]. 1-Aminocyclopropane-1-carboxylate is the direct precursor of the plant hormone ethylene and plays a role in the regulation of plant development [120]. L-Allothreonine is a stereoisomer of threonine and an intermediate in plant glycine, serine, and threonine metabolism [121]. 3-Phenoxybenzyl alcohol is a mammalian metabolite of the insecticide permethrin produced by carboxylesterases [122,123]. Dihydroshikonofuran is a monoterpenoid product of the ubiquinone biosynthetic pathway that also produces shikonin, a plant pigment with anti-inflammatory, antibacterial, and wound-healing properties $[124,125]$. Phenethyl alcohol is a natural fragrance produced by rose, carnation, and other plants $[126,127]$.

\section{Limitations}

While this analysis provides a comprehensive scan of potential metabolic targets in dogs receiving CBD, it was not intended to be all-encompassing. Because of the lack of metabolic profile with CBD, this was intended to be an exploratory first look into the potential for CBD supplementation to alter the canine metabolome. Limitations of this study include the relatively small sample size, lack of baseline measurement, short duration of CBD supplementation, and the use of only a single dose $(\mathrm{mg} / \mathrm{kg})$ of CBD. Additionally, as neither the treats nor CBD extract were analyzed, it is possible that the increased metabolites were present as a result of CBD extract supplementation rather than an alteration of metabolism. Even so, identifying changes in the metabolome is essential for directing future targeted investigations into both the physiological relevance of these changes as well as elucidating potential mechanisms leading to these observed effects. It would be beneficial for future work to evaluate metabolomic changes in an unhealthy or diseased population of dogs supplemented with $\mathrm{CBD}$ and to investigate potential differences between short- and long-term CBD administration.

\section{Conclusions}

This analysis showed an alteration of canine carboxyl and hydroxyl submetabolomes after 3 weeks of $4 \mathrm{mg} \mathrm{CBD} / \mathrm{kg}$ BW/d supplementation. Altered metabolites may suggest a potential for CBD to influence lipid, carbohydrate, amino acid, vitamin, and nucleotide metabolism as well as possible links with the host mcrobiome. Several metabolites were identified as potential biomarkers for changes in the canine metabolome by CBD that may be used in future work using a targeted metabolomics approach. Additionally, changes in relative concentrations of metabolites like acetic acid, 9-oxononanoic acid, and 3,4-dihydroxymandelic acid may indicate potential pathways by which CBD may exert suspected anti-inflammatory, antioxidant, and anti-obesity effects. Future work would benefit from larger sample sizes, longer supplementation periods, and multiple CBD doses.

\section{Author Contributions}

$\mathrm{DH}, \mathrm{EM}, \mathrm{KM}$, and SK contributed to the conception and design of the study. EM, SK, and DS facilitated data collection. EM and IO performed statistical analysis. EM wrote the first draft of the manuscript. All authors contributed to manuscript revision, read, and approved the submitted version.

\section{Funding}

The authors declare that this study received funding from AgTech Scientific, Paris, KY. The funder was not involved in the study design, collection, analysis, interpretation of data, the writing of this article, or the venue for publication.

\section{Acknowledgements}

We thank Lincoln Memorial University students $\mathrm{K}$ Athey, $\mathrm{H}$ Barnhart, L Calvin, K Dubois, J Gauldin, S Swears, M Kight, M Mendoza, J Steen, S Swears, and K Williams for their assistance in caring for dogs and facilitating data collection.

\section{References}

1. Landa L, Sulcova A, Gbelec P (2016) The use of cannabinoids in animals and therapeutic implications for veterinary medicine: a review. Veterinární Medicína 61: 111-122.

2. Ogunade IM, Jiang $\mathrm{Y}$, Adeyemi J, Oliveira A, Vyas $D$, et al. (2018) Biomarker of aflatoxin ingestion: ${ }^{1} \mathrm{H}$ NMR-based plasma metabolomics of dairy cows fed aflatoxin $B_{1}$ with or without sequestering agents. Toxins (Basel) 10: 545.

3. Yang Y, Dong G, Wang Z, Wang J, Zhang Z, et al. (2018) Rumen and plasma metabolomics profiling by UHPLC-QTOF/MS revealed metabolic alterations associated with a high-corn diet in beef steers. PLOS ONE 13: e0208031.

4. Ogunade I, Oyebade A, Osa-Andrews B, Peters S (2021) Plasma carboxyl-metabolome is associated with average daily gain divergence in beef steers. Animals (Basel) 11: 67.

5. Zhao S, Luo X, Li L (2016) Chemical isotope labeling LC-MS for high coverage and quantitative profiling of the hydroxyl submetabolome in metabolomics. Anal Chem 88: 10617-10623.

6. Morris EM, Kitts-Morgan SE, Spangler DM, Ogunade IM, McLeod KR, et al. (2021a) Alteration of the canine metabolome after a threeweek supplementation of cannabidiol (CBD) containing treats: An exploratory study of healthy animals. Front Vet Sci 8: 685606.

7. Bielawiec P, Harasim-Symbor E, Chabowski A (2020) Phytocannabinoids: Useful drugs for the treatment of obesity? Special focus on cannabidiol. Front Endocrinol 11: 114.

8. Morris EM, Kitts-Morgan SE, Spangler DM, Gebert J, Vanzant ES, et al. (2021b) Feeding cannabidiol (CBD) containing treats did not affect canine daily voluntary activity. Front Vet Sci 8: 645667.

9. Mahajan DK, Anderson G, Poole WK, Billar RB, Little B (1983) Changes in the concentration of 17 alpha,20 alpha-dihydroxypregn4-en-3-one during pregnancy, labor, and delivery and the effect of dexamethasone treatment during the third trimester of pregnancy. J Clin Endocrinol Metab 57: 585-591.

10. Shikita M, Inano H, Tamaoki B (1967) Further studies on 20-alphahydroxysteroid dehydrogenase of rat testes. Biochemistry 6: 17601764.

11. Tiranti V, D'Adamo P, Briem E, Ferrari G, Mineri R, et al. (2004) Ethylmalonic encephalopathy is caused by mutations in ETHE1, a gene encoding a mitochondrial matrix protein. Am J Hum Genet 74: 239-252.

12. Wolfe L, Jethva R, Oglesbee D, Vockley J (2011) Short-chain acylCoA dehydrogenase deficiency. In: Adam MP, Ardinger HH, Pagon RA, Wallace SE, Bean LJH, Mirzaa G, Amemiya A.(eds) Gene Reviews (Seattle, WA: University of Washington, Seattle) 1993-2021.

13. Wang X, Zhao X, Chou J, Yu J, Yang T, et al. (2018) Taurine, glutamic acid and ethylmalonic acid as important metabolites for detecting human breast cancer based on the targeted metabolomics. Cancer Biomark 23: 255-268.

14. Miyazaki T, Honda A, Ikegami T, Iwamoto J, Monma $T$, et al. (2015) Simultaneous quantification of salivary 3-hydroxybutyrate, 3-hydroxyisobutyrate, 3-hydroxy-3-methylbutyrate, and 2-hydroxybutyrate as possible markers of amino acid and fatty acid catabolic pathways by LC-ESI-MS/MS. Springer plus 4: 494. 
15. Den H, RobinsonWG, Coon MJ (1959) Enzymatic conversion of beta-hydroxypropionate to malonic semialdehyde. J Biol Chem 234: 1666-1671.

16. Hawes JW, Jaskiewicz J, Shimomura Y, Huang B, Bunting J, et al. (1996) Primary structure and tissue-specific expression of human beta-hydroxyisobutyryl-coenzyme A hydrolase. J Biol Chem 271: 26430-26434.

17. Louis P, Flint HJ (2009) Diversity, metabolism and microbial ecology of butyrate-producing bacteria from the human large intestine. FEMS Microbiol Lett 294: 1-8.

18. Macfarlane S, Macfarlane GT (2003) Regulation of short-chain fatty acid production. Proc Nutr Soc 62: 67-72.

19. Marcal D, Rego AT, Carrondo MA, Enguita FJ (2009) 1,3-Propanediol dehydrogenase from Klebsiella pneumoniae: Decameric quaternary structure and possible subunit cooperativity. J Bacteriol 191: 11431151.

20. Blötz C, Stülke J (2017) Glycerol metabolism and its implication in virulence in Mycoplasma. FEMS Microbiol Rev 41: 640-652.

21. Jin ES, Browning JD, Murphy RE, Malloy CR (2018) Fatty liver disrupts glycerol metabolism in gluconeogenic and lipogenic pathways in humans. J Lipid Res 59: 1685-1694.

22. Mahendran $\mathrm{Y}$, Cederberg $\mathrm{H}$, Vangipurapu J, Kangas $\mathrm{AJ}$, Soininen $\mathrm{P}$, et al. (2013) Glycerol and fatty acids in serum predict the development of hyperglycemia and type 2 diabetes in Finnish men. Diabetes Care 36: 3732-3738.

23. Braggelaar MP, Maccarrone M, Van der Stelt M (2018) 2-Arachidonoylglycerol: A signaling lipid with manifold actions in the brain. Prog Lipid Res 71: 1-17.

24. Giang DK, Cravatt BF (1997) Molecular characterization of human and mouse fatty acid amide hydrolase. Proc Natl Acad Sci USA 94 2238-2242.

25. Elmes MW, Kaczocha M, Berger WT, Leung K, Ralph BP, et al. (2015) Fatty acid-binding proteins (FABPs) are intracellular carriers for $\Delta 9$ tetrahydrocannabinol (THC) and cannabidiol (CBD). J Biol Chem 290: 8711-8721.

26. Leweke FM, Piomelli D, Pahlisch F, Muhl D, Gerth CW, et al. (2012) Cannabidiol enhances anadamide signaling and alleviates psychotic symptoms of schizophrenia. Transl Psychiatry 2: e94.

27. Schroeder F, McIntosh AL, Martin GG, Huang $H$, Landrock $D$, et al. (2016) Fatty acid binding protein-1 (FABP1) and the human FABP1 T94A variant: Roles in the endocannabinoid system and dyslipidemias. Lipids 51: 655-676.

28. Otte KB, Kirtz M, Nestl BM, Hauer B (2013) Synthesis of 9-oxonanonic acid, a precursor for biopolymers. ChemSusChem 6: 21492156.

29. Kanazawa K, Ashida H, Minamoto S, Natake M (1986) The effect of orally administered secondary autoxidation products of linoleic acid on the activity of detoxifying enzymes in the rat liver. Biochim Biophys Acta 879: 36-43.

30. Minamoto S, Kanazawa K, Ashida H, Natake M (1988) Effect of orally administered 9-oxononanoic acid on lipogenesis in rat liver. Biochim Biophys Acta 958: 199-204.

31. Ren $R$, Hashimoto $T$, Mizuno $M$, Takigawa $H$, Yoshida $M$, et al. (2013) A lipid peroxidation product 9-oxononanoic acid induces phospholipase A2 activity and thromboxane A2 production in human blood. J Clin Biochem Nutr 52: 228-233.
32. Behr M, Pokorna E, Dobrev PI, Motyka V, Guignard C, et al. (2019) Impact of jasmonic acid on lignification in the hemp hypocotyl. Plant Signal Behav 14: 1592641.

33. Henriet E, Jager S, Tran C, Bastien P, Michelet J-F, et al. (2017) A jasmonic acid derivative improves skin healing and induces changes in proteoglycan expression and glycosaminoglycan structure. Biochim Biophys Acta Gen Subj 1861: 2250-2260.

34. Falck JR, Reddy LM, Byun K, Campbell WB, Yi X-Y (2007) Epoxygenase eicosanoids: synthesis of tetrahydrofuran-diol metabolites and their vasoactivity. Bioorg Med Chem Lett 17: 2634-2638.

35. Reddy DS (2006) Physiological role of adrenal deoxycorticosteronederived neuroactive steroids in stress-sensitive conditions. Neuroscience 138: 911-920.

36. Reddy DS, Rogawski MA (2002) Stress-induced deoxycorticosteronederived neurosteroids modulate $\mathrm{GABA}(\mathrm{A})$ receptor function and seizure susceptibility. J Neurosci 22: 3795-3805.

37. Kornel L, Patel SK, Ezzeraimi E, Shackleton CH (1995) Corticosteroids in human blood: IX. Evidence for adrenal secretion of sulfateconjugated cortisol, 11 beta,17 alpha-dihydroxy-4-pregnene-3,20dione-21-yl-sulfate. Steroids 60: 817-823.

38. Hosoda H, Yokohama H, Nambara T (1984) Synthesis of cortol 3-glucuronides and cortolone 3-glucuronides. Chem Pharm Bull (Tokyo) 32: 4023-4028.

39. Yang G, Ge S, Singh R, Basu S, Shatzer K, et al. (2017) Glucuronidation: driving factors and their impact on glucuronide disposition. Drug Metab Rev 49: 105-138.

40. Katz J, Tayek JA (1998) Gluconeogenesis and the Cori cycle in 1220-, and 40-h-fasted humans. Am J Physiol 275: E537-E542.

41. Nuttall FQ, Ngo A, Gannon MC (2008) Regulation of hepatic glucose production and the role of gluconeogenesis in humans: Is the rate of gluconeogenesis constant? Diabetes Metab Res Rev 24: 438-458.

42. Dawkins PD, Dickens F (1965) The oxidation of D- and L-glycerate by rat liver. Biochem J 94: 353-367.

43. Heinz F, Lamprecht W, Kirsch J (1968) Enzymes of fructose metabolism in human liver. J Clin Invest 47: 1826-1832.

44. Mu W, Hassanin HAM, Zhou L, Jiang B (2018) Chemistry behind rare sugars and bioprocessing. J Agric Food Chem 66: 13343-13345.

45. Bohren KM, Bullock B, Wermuth B, Gabbay KH (1989) The aldo-keto reductase superfamily. cDNAs and deduced amino acid sequences of human aldehyde and aldose reductases. J Biol Chem 264: 95479551.

46. Saracyn M, Zdanowski R, Brytan M, Kade G, Nowak Z, et al. (2015) D-galactosamine intoxication in experimental animals: Is it only an experimental model of acute liver failure? Med Sci Monit 21: 14691477.

47. Wu Y-H, Hu S-Q, Liu J, Cao H-C, Xu W, et al. (2014) Nature and mechanisms of hepatocyte apoptosis induced by D-glalactosamine/ lipopolysaccharide challenge in mice. Int J Mol Med 33: 1498-1506.

48. Becker DJ, Lowe JB (2003) Fucose: Biosynthesis and biological function in mammals. Glycobiology 13: 41R-53R.

49. Rigo LU, Marechal LR, Vieira MM, Veiga LA (1985) Oxidative pathway for L-rhamnose degredation in Pullularia pullulans. Can J Microbiol 31: 817-822.

50. Wen L, Zang L, Huang K, Li S, Wang R, et al. (2016) Efficient enzymatic synthesis of L-rhamnulose and L-fuculose. Bioorg Med Chem Lett 26: 969-972. 
51. Vanhooren PT, Vandamme EJ (1999) L-fucose: Occurrence, physiological role, chemical, enzymatic and microbial synthesis. Chem Technol Biotechnol 74: 479-497.

52. Hong HA, Khaneja R, Tam NMK, Cazzato A, Tan S, et al. (2009) Bacillus subtilis isolated from the human gastrointestinal tract. Res Microbiol 160: 134-143.

53. Yoshida K-I, Yamaguchi M, Morinaga T, Kinehara M, Ikeuchi M, et al. (2008) myo-Inositol catabolism in Bacillus subtilis. J Biol Chem 283: 10415-10424.

54. Kordowska-Wiater M (2015) Production of arabitol by yeasts: Current status and future prospects. J Appl Microbiol 119: 303-314.

55. Salonen JH, Rimpilainen $\mathrm{M}$, Lehtonen L, Lehtonen OP, Nikoskelainen $J$ (2001) Measurement of the $D$-arabinitol/L-arabinitol ratio in urine of neutropenic patients treated empirically with amphotericin $B$. Eur J Clin Microbiol Infect Dis 20: 179-184.

56. Verhoeven NM, Huck JH, Roos B, Struys EA, Salomons GS, et al. (2001) Transaldolase deficiency: Liver cirrhosis associated with a new in born error in the pentose phosphate pathway. Am J Hum Genet 68: 1086-1092.

57. Zhu G, Yin C, Tian Z, WangT, Sun W, et al. (2015) Metabolomic analysis of plasma from patients with acute mountain sickness using chromatography-mass spectrometry. Medicine (Baltimore) 94: e1988.

58. McCabe M, Cital S (2021) Cannabinoids for Gastrointestinal Health. In: Cital S, Kramer K, Hughston L, Gaynor JS, Cannabis Therapy in Veterinary Medicine. Springer $1^{\text {st }}$ Edition.

59. Watanabe $S$, Morimoto D, Fukumori F, Shinomiya $H$, Nishiwaki $H$, et al. (2012) Identification and characterization of D-hydroxyproline dehydrogenase and Delta1-pyrroline-4-hydroxy-2-carboxylate deaminase involved in novel L-hydroxyproline metabolism of bacteria: metabolic convergent evolution. J Biol Chem 287: 32674 32688 .

60. Brouns SJJ, Walther J, Snijders APL, Van de Werken HJG, Willemen HLDM, et al. (2006) Identification of the missing links in prokaryotic pentose oxidation pathways: Evidence for enzyme recruitment. J Biol Chem 281: 27378-27388.

61. Johnsen U, Dambeck M, Zaiss H, Fuhrer T, Soppa J, et al. (2009) D-xylose degradation pathway in the halophilic archaeon Haloferax volcanii. J Biol Chem 284: 27290-27303.

62. Watanabe S, Shimada N, Tajima K, Kodaki T, Makino K (2006) Identification and characterization of L-arabonate dehydratase, L-2-keto-3-deoxyarabonate dehydratase, and L-arabinolactonase involved in an alternative pathway of L-arabinose metabolism. Novel evolutionary insight into sugar metabolism. J Biol Chem 281: 33521-33536.

63. Parimoo B, Tanaka T (1993) Structural organization of the human isovaleryl-CoA dehydrogenase gene. Genomics 15: 582-590.

64. Bellinzoni $M$, Bastard $K$, Perret $A$, Zaparucha $A$, Perchat $N$, et al. (2011) 3- Keto-5-aminohexanoate cleavage enzyme: a common fold for an uncommon Claisen-type condensation. J Biol Chem 286: 27399-27405.

65. Kreimeyer A, Perret A, Lechaplais C, Vallenet D, Medigue C, et al (2007) Identification of the last unknown genes in the fermentation pathway of lysine. J Biol Chem 282: 7191-7197.

66. Visser WF, Verhoeven-Duif NM, de Koning TJ (2012) Identification of a human trans-3-hydroxy-L-proline dehydratase, the first characterized member of a novel family of proline racemase-like enzymes. J Biol Chem 287: 21654-21662.
67. Bartsch O, Hagemann M, Bauwe H (2008) Only plant-type (GLYK) glycerate kinases produce d-glycerate 3-phosphate. FEBS Lett 582: 3025-3028.

68. Randall DD, Tolbert NE (1971) 3-Phosphoglycerate phosphatase in plants. I. Isolation and characterization from sugarcane leaves. J Biol Chem 246: 5510-5517.

69. Guo J-H, Hexige S, Chen L, Zhou G-J, Wang X, et al. (2006) Isolation and characterization of the human D-glyceric acidemia related glycerate kinase gene GLYCTK1 and its alternatively splicing variant GLYCTK2. DNA Seq 17: 1-7.

70. Sass JO, Fischer K, Wang R, Christensen E, Scholl-Burgi S, et al. (2010) $\mathrm{D}$-glyceric aciduria is caused by genetic deficiency of D-glycerate kinase (GLYCTK). Hum Mutat 31: 1280-1285.

71. Kisaki T, Tolbert NE (1969) Glycolate and glyoxylate metabolism by isolated peroxisomes or chloroplasts. Plant Physiol 44: 242-250.

72. Belostotsky R, Pitt JJ, Frishberg Y (2012) Primary hyperoxaluria type III-a model for studying perturbations in glyoxylate metabolism. J Mol Med (Berl) 90: 1497-1504.

73. Salido E, Pey AL, Rodriguez R, Lorenzo V (2012) Primary hyperoxalurias: Disorders of glyoxylate detoxification. Biochim Biophys Acta 1822: 1453-1464.

74. Davis WL, Goodman DB (1992) Evidence for the glyoxylate cycle in human liver. Anat Rec 234: 461-468.

75. Song $S$ (2000) Can the glyoxylate pathway contribute to fat-induced hepatic insulin resistance? Med Hypotheses 54: 739-747.

76. Nikiforova VJ, Giesbertz P, Wiemer J, Bethan B, Looser R, et al. (2014) Glyoxylate, a new marker metabolite of type 2 diabetes. J Diabetes Res 2014: 685204.

77. Padberg I, Peter E, Gonzalez-Maldonado S, Witt H, Mueller M, et al (2014) A new metabolic signature in type-2 diabetes mellitus and its pathophysiology. PLoS One 9: e85082.

78. Barkulis SS, Krakow G (1956) Conversion of glyoxylate to hydroxypyruvate by extracts of Escherichia coli. Biochim Biophys Acta 21: 593-594.

79. Gupta NK, Vennesland B (1964) Glyoxylate carboligase of Escherichia coli: A flavoprotein. J Biol Chem 239: 3787-3789.

80. Vella GJ, Hill RE, Mootoo BS, Spenser ID (1980) The status of glycolaldehyde in the biosynthesis of vitamin B6. J Biol Chem 255: 3042-3048.

81. Ley JP, Engelhart K, Bernhardt J, Bertram H-J (2002) 3,4-Dihydroxymandelic acid, a noradrenalin metabolite with powerful antioxidative potential. J Agric Food Chem 50: 5897-5902.

82. Podebrad F, Heil M, Beck T, Mosandl A, Sewell AC, et al. (2000) Stereodifferentiation of 3-hydroxyisobutyric- and 3-aminoisobutyric acid in human urine by enantioselective multidimensional capillary gas chromatography-mass spectrometry. Clin Chim Acta 292: 93105.

83. O'Kell AL, Garrett TJ, Wasserfall C, Atkinson MA (2017) Untargeted metabolomic analysis in naturally occurring canine diabetes mellitus identifies similarities to human Type 1 Diabetes. Sci Rep 7: 9467.

84. Abaskharon RM, Mukherjee D, Gai F (2019) 4-Oxoproline as a SiteSpecific Infrared Probe: Application to Assess Proline Isomerization and Dimer Formation. J Phys Chem B 123: 5079-5085.

85. Hu CA, Lin WW, Valle D (1996) Cloning, characterization, and expression of cDNAs encoding human delta 1-pyrroline-5carboxylate dehydrogenase. J Biol Chem 271: 9795-9800. 
86. Watanabe S, Tajima K, Fujii S, Fukumori F, Hara R, et al. (2016) Functional characterization of aconitase $\mathrm{X}$ as a cis-3-hydroxy-Lproline dehydratase. Sci Rep 6: 38720.

87. Durg S, Lobo M, Venkatachalam L, Rao G, Bhate J (2019) A systematic review and meta-analysis of oxaceprol in the management of osteoarthritis: An evidence from randomized parallel-group controlled trials. Pharmacol Rep 71: 374-383.

88. Colabroy KL, Begley TP (2005) Tryptophan catabolism: identification and characterization of a new degradative pathway. J Bacteriol 187: 7866-7869.

89. Nishizuka Y, Ichiyama A, Gholson RK, Hayaishi O (1965) Studies on the Metabolism of the Benzene Ring of Tryptophan in Mammalian Tissues: I. Enzymic formation of glutaric acid from 3-hydroxy anthranilic acid. J Biol Chem 240: 733-739.

90. Cervenka I, Agudelo LZ, Ruas JL (2017) Kynurenines: Tryptophan's metabolites in exercise, inflammation, and mental health. Science 357: eaaf9794.

91. Thiele I, Swainston N, Fleming RMT, Hoppe A, Sahoo S, et al. (2013) A community-driven global reconstruction of human metabolism. Nat Biotechnol 31: 419-425.

92. Ma Q, Zhang X, Qu Y (2018) Biodegradation and biotransformation of indole: advances and perspectives. Front Microbiol 9: 2625

93. Koketsu K, Mitsuhashi S, Tabata K (2013) Identification of homophenylalanine biosynthetic genes from the cyanobacterium Nostoc punctiforme PCC73102 and application to its microbial production by Escherichia coli. Appl Environ Microbiol 79: 2201 2208.

94. Land EJ, Ramsden CA, Riley PA (2003) Tyrosinase autoactivation and the chemistry of ortho-quinone amines. Acc Chem Res 36: 300-308.

95. Olivares C, Jimenez-Cervantes C, Lozano JA, Solano F, Garcia-Borron JC (2001) The 5,6-dihydroxyindole-2-carboxylic acid (DHICA) oxidase activity of human tyrosinase. Biochem J 354: 131-139.

96. Kang SS, Mun K-C, Seo JH, Choe M, Ha E (2018) Cyanate improves insulin sensitivity and hepatic steatosis in normal and high fat-fed mice: Anorexic and antioxidative effects. Chem Biol Interact 279: 121-128.

97. Kwack MH, Ahn JS, Kim MK, Kim JC, Sung YK (2010) Preventable effect of L-threonate, an ascorbate metabolite, on androgen-driven balding via repression of dihydrotestosterone-induced dickkopf-1 expression in human hair dermal papilla cells. BMB Rep 43: 688-692.

98. Sun Q, Weinger JG, Mao F, Liu G (2016) Regulation of structural and functional synapse density by L-threonate through modulation of intraneuronal magnesium concentration. Neuropharmacology 108: 426-439.

99. Wang H, Hu P, Jiang J (2011) Pharmacokinetics and safety of calcium L-threonate in healthy volunteers after single and multiple oral administrations. Acta Pharmacol Sin 32: 1555-1560.

100. Kirkland JB (2009) Niacin status, NAD distribution and ADP-ribose metabolism. Curr Pharm. Des 15: 3-11.

101. Krehl WA, Torbet N, de la Huerga J, Elvehjem CA (1946) Relation of synthetic folic acid to niacin deficiency in dogs. Arch Biochem 11: 363-369.

102. Scholem RD, Brown GK (1983) Metabolism of malonic semialdehyde in man. Biochem J 216: 81-85.

103. Bradford KL, Moretti FA, Carbonaro-Sarracino DA, Gaspar HB, Kohn DB (2017) Adenosine deaminase (ADA)-Deficient Severe Combined Immune Deficiency (SCID): Molecular pathogenesis and clinical manifestations. J Clin Immunol 37: 626-637.
104. Gray MJ, Escalante-Semerena JC (2010) A new pathway for the synthesis of $\alpha$-ribazole-phosphate in Listeria innocua. Mol Microbiol 77: 1429-1438.

105. Pakin C, Bergaentzle M, Aoude-Werner D, Hasselmanna C (2005) Alpha-ribazole, a fluorescent marker for the liquid chromatographic determination of vitamin B12 in foodstuffs. J Chromatogr A 1081: 182-189.

106. Li X, Yu C, Cai Y, Liu G, Jia J, et al. (2005) Simultaneous determination of six phenolic constituents of danshen in human serum using liquid chromatography/tandem mass spectrometry. J Chromatogr B Analyt Technol Biomed Life Sci 820: 41-47.

107. Schneider K, Kienow L, Schmelzer E, Colby T, Bartsch M, et al. (2005) A new type of peroxisomal acyl-coenzyme A synthetase from Arabidopsis thaliana has the catalytic capacity to activate biosynthetic precursors of jasmonic acid. J Biol Chem 280: 1396213972.

108. Ranjith NK, SasikalaC, Ramana CV (2007) Catabolism of L-phenylalanine and L-tyrosine by Rhodobacter sphaeroides OU5 occurs through 3,4-dihydroxyphenylalanine. Res Microbiol 158: 506-511.

109. Wang J, Hodes GE, Zhang H, Zhang S, Zhao W, et al. (2018) Epigenetic modulation of inflammation and synaptic plasticity promotes resilience against stress in mice. Nat Commun 9: 477.

110. Panoutsopoulos GI, Beedham C (2005) Metabolism of isovanillin by aldehyde oxidase, xanthine oxidase, aldehyde dehydrogenase and liver slices. Pharmacology 73: 199-208.

111. Strand LP, Scheline RR (1975) The metabolism of vanillin and isovanillin in the rat. Xenobiotica 5: 49-63.

112. Loke WM, Jenner AM, Proudfoot JM, McKinley AJ, Hodgson JM, et al. (2009) A metabolite profiling approach to identify biomarkers of flavonoid intake in humans. J Nutr 139: 2309-2314.

113. Zarzycki J, Brecht V, Muller M, Fuchs G (2009) Identifying the missing steps of the autotrophic 3-hydroxypropionate $\mathrm{CO}_{2}$ fixation cycle in Chloroflexus aurantiacus. Proc Natl Acad Sci USA 106: 21317-21322.

114. Shaw W, Kassen E, Chaves E (1995) Increased urinary excretion of analogs of Krebs cycle metabolites and arabinose in two brothers with autistic features. Clin Chem 41: 1094-1104.

115. Smith N, Longo N, Levert K, Hyland K, Blau N (2019) Phase I clinical evaluation of CNSA-001 (sepiapterin), a novel pharmacological treatment for phenylketonuria and tetrahydrobiopterin deficiencies, in healthy volunteers. Mol Genet Metab 126: 406-412.

116. Wang H, Yang B, Hao G, Feng $Y$, Chen H, et al. (2011) Biochemical characterization of the tetrahydrobiopterin synthesis pathway in the oleaginous fungus Mortierella alpine. Microbiology (Reading) 157: 3059-3070.

117. Tarpey MM (2002) Sepiapterin treatment in atherosclerosis. Arterioscler Thromb Vasc Biol 22: 1519-1521.

118. Vásquez-Vivar J, Duquaine D, Whitsett J, Kalyanaraman B, Rajagopalan S (2002) Altered tetrahydrobiopterin metabolism in atherosclerosis: implications for use of oxidized tetrahydrobiopterin analogues and thiol antioxidants. Arterioscler Thromb Vasc Biol 22: 1655-1661.

119. Campanini B, Bettati S, di Salvo ML, Mozzarelli A, Constestabile R (2013) Asymmetry of the active site loop conformation between subunits of glutamate-1-semialdehyde aminomutase in solution. Biomed Res Int 2013: 353270. 
120. Polko JK, Kieber JJ (2019) 1-Aminocyclopropane 1-carboxylic acid and its emerging role as an ethylene-independent growth regulator. Front Plant Sci 10: 1602.

121. Fiehn O, Kopka J, Trethewey RN, Willmitzer L (2000) Identification of uncommon plant metabolites based on calculation of elemental compositions using gas chromatography and quadrupole mass spectrometry. Anal Chem 72: 3573-3580.

122. Crow JA, Borazjani A, Potter PM, Ross MK (2007) Hydrolysis of pyrethroids by human and rat tissues: examination of intestinal, liver and serum carboxylesterases. Toxicol Appl Pharmacol 221: 1-12.

123. Stok JE, Huang H, Jones PD, Wheelock CE Morisseau C, et al. (2004) Identification, expression, and purification of a pyrethroidhydrolyzing carboxylesterase from mouse liver microsomes. J Biol Chem 279: 29863-29869.
124. Boehm R, Sommer S, Li SM, Heide L (2000) Genetic engineering on shikonin biosynthesis: expression of the bacterial ubiA gene in Lithospermum erythrorhizon. Plant Cell Physiol 41: 911-919.

125. Yazaki K, Fukui H, Tabata M (1987) Dihydroshikonofuran, an unuaul metabolite of quinone biosynthesis in Lithospermum cell cultures. Chem Pharm Bull 35: 898-901.

126. Guterman I, Masci T, Chen X, Negre F, Pichersky E, et al. (2006) Generation of phenylpropanoid pathway-derived volatiles in transgenic plants: rose alcohol acetyltransferase produces phenylethyl acetate and benzyl acetate in petunia flowers. Plant Mol Biol 60: 555-563.

127. Politano VT, Diener RM, Christian MS, Hawkins DR, Ritacco G, et al. (2013) The pharmacokinetics of phenylethyl alcohol (PEA): safety evaluation comparisons in rats, rabbits, and humans. Int J Toxicol 32: $39-47$ 